\title{
OPEN Effects of the antibiotic rifaximin on cortical functional connectivity are mediated through insular cortex
}

\author{
Davide Sometti ${ }^{1,2,3 凶}$, Chiara Ballan ${ }^{1,3}$, Huiying Wang ${ }^{4}$, Christoph Braun ${ }^{1,2,3,5}$ \& Paul Enck ${ }^{6}$
}

It is well-known that antibiotics affect commensal gut bacteria; however, only recently evidence accumulated that gut microbiota (GM) can influence the central nervous system functions. Preclinical animal studies have repeatedly highlighted the effects of antibiotics on brain activity; however, translational studies in humans are still missing. Here, we present a randomized, double-blind, placebo-controlled study investigating the effects of 7 days intake of Rifaximin (non-absorbable antibiotic) on functional brain connectivity (fc) using magnetoencephalography. Sixteen healthy volunteers were tested before and after the treatment, during resting state ( $r s$ ), and during a social stressor paradigm (Cyberball game-CBG), designed to elicit feelings of exclusion. Results confirm the hypothesis of an involvement of the insular cortex as a common node of different functional networks, thus suggesting its potential role as a central mediator of cortical fc alterations, following modifications of GM. Also, the Rifaximin group displayed lower connectivity in slow and fast beta bands (15 and $25 \mathrm{~Hz}$ ) during rest, and higher connectivity in theta $(7 \mathrm{~Hz})$ during the inclusion condition of the CBG, compared with controls. Altogether these results indicate a modulation of Rifaximin on frequency-specific functional connectivity that could involve cognitive flexibility and memory processing.

The interest in human gut microbiota and its effects on CNS functioning increased exponentially over the last decade $^{1-9}$. Strong preclinical evidence testified an effect of the GM on brain functioning and development ${ }^{10}$. Following the pioneering study of Sudo and colleague ${ }^{11}$ reporting an alteration of the hypothalamic-pituitary-adrenal (HPA) stress response in germ-free mice, data have accumulated confirming the role of the commensal microbiota in stress responsiveness ${ }^{12-15}$, anxiety and depression-like behaviors, and cognitive functions ${ }^{16-19}$. However, when studies are translated into humans, understanding the effects of GM on CNS becomes more difficult ${ }^{20}$. Significant ethical constraints and several environmental factors such as $\operatorname{diet}^{21,22}$, psychological stress ${ }^{23}$, tobacco and alcohol consumption ${ }^{24,25}$ limit the causational interpretation of the interaction between GM and brain functioning. Nevertheless, the approach of targeting human $\mathrm{GM}^{26-28}$ has offered a potential method to investigate its effect on the CNS.

Starting from the idea of a possible application of probiotics as adjuvant therapy for the treatment of the major depressive disorder ${ }^{29}$, the curiosity in the effects of some specific "Psychobiotic" ${ }^{30}$ influencing the functionality of the central nervous system has grown rapidly ${ }^{31-35}$. Clinical studies reported alteration in cognitive performance and self-reported measures of stress, anxiety, and depression-like behavior ${ }^{36-39}$, following Lactobacillus and Bifidobacterium strains intake in healthy volunteers. Neuroimaging methods offered a deeper understanding of the effects of probiotics on brain activity. Tillisch and colleagues ${ }^{40}$ demonstrated that 4 -weeks consumption of a fermented milk product containing probiotics altered the activation of interoceptive and somatosensory regions, together with rs midbrain connectivity, in response to an emotional attention fMRI task. Reduced limbic reactivity to fearful faces was found in patients with Irritable Bowel Syndrome (IBS) after 6-weeks of treatment with a Bifidobacterium strain ${ }^{41}$. Other neuroimaging studies reported alteration in brain activity associated with emotional decision-making and memory processes ${ }^{42}$, rs functional connectivity ${ }^{43}$, and stress-related

${ }^{1}$ MEG-Center, University of Tübingen, Tübingen, Germany. ${ }^{2}$ Hertie Institute for Clinical Brain Research, University of Tübingen, Tübingen, Germany. ${ }^{3}$ DiPSCo, Department of Psychology and Cognitive Science, University of Trento, Rovereto, Italy. ${ }^{4} \mathrm{AAK}$, Department of Special Nutrition, AAK China Ltd, Shanghai, China. ${ }^{5} \mathrm{CIMeC}$, Center for Mind/Brain Research, University of Trento, Trento, Italy. ${ }^{6}$ Department of Internal Medicine VI, University Hospital, Tübingen, Germany. ${ }^{\varpi}$ email: davide.sometti-1@alumni.unitn.it 
electrophysiology ${ }^{44,45}$, following Bifidobacterium and Lactobacillus intake by healthy volunteers. Nonetheless, despite these encouraging results, not all potential probiotic interventions that had shown an effect on CNS in preclinical studies have translated successfully in humans ${ }^{46}$. Thus, highlighting the need for a more comprehensive understanding of the role played by the intestinal microbiota in altering brain activity.

A complementary approach to the study of GM-fc interaction by administering probiotics is offered by antibiotics, which typically causes a decrease in microbiota diversity ${ }^{47-49}$. Preclinical studies have repeatedly shown the impact of antibiotic-induced GM depletion on stress-related behavior and cognitive functions in rodents ${ }^{50-54}$. Concerning human studies on GM, antibiotics have almost exclusively been studied to test their efficacy in treating gastrointestinal (GI) diseases ${ }^{55,56}$. Among the different antibiotics employed, rifaximin has been proven effective in treating small intestine bacterial overgrowth (SIBIO-review ${ }^{57}$ ) and GI diseases such as traveler's diarrhea, functional bloating, and irritable bowel syndrome (IBS). Furthermore, due to its negligible systemic absorption after oral administration (less than $0.4 \%$, reviews ${ }^{58,59}$ ), the risk of developing drug resistance in healthy subjects is minimized ${ }^{60-63}$. For these reasons, Rifaximin has been recently selected by our group to first examining the effects of antibiotics on CNS in healthy volunteers ${ }^{64}$. The study investigated the effects of 7-weeks Rifaximin intake on brain activity with MEG. Results for antibiotic treatment displayed an increase of self-reported emotional well-being, together with an alteration in resting-state alpha and task-related beta power associated with a stress-reducing effect. These first findings exhibited an effect of Rifaximin on brain activity similar to the one exerted by the intake of probiotics ${ }^{45}$. Nevertheless, whether Rifaximin may modulate intracortical coupling, thus altering functional connectivity, is yet unknown.

Herein, we aimed at expanding our previous findings on spectral power investigating the effect of 7-days Rifaximin administration on MEG functional connectivity in healthy subjects. Brain connectivity was investigated during rest and during a social stress-inducing paradigm (Cyberball game-CBG) eliciting feelings of ostracism. We anticipate the involvement of the insula in signalling Rifaximin effects to the cortex, due to its critical role in interoception ${ }^{65,66}$. Considering the presence of visceral afferent projections that from the vagus nerve reach the insular cortex through the thalamus, the insula seems a plausible candidate to be a significant hub within the microbiota-gut-brain axis. Given the pilot nature of the study, no preconceived assumptions regarding the different frequencies of coherence that might be affected by the treatment, were present. Inclusion criteria, group characteristics, and experimental paradigm have already been reported in detail ${ }^{64}$ and will not be reported here unless necessary for the understanding of the current approach.

\section{Results}

Effects of rifaximin on resting-state connectivity. To check the presence of significant differences regardless of the treatment, we compared the groups at the baseline. No differences were found in any frequency band. After 7 -days treatment, the placebo group displayed networks with higher fc in slow-beta $(15 \mathrm{~Hz}$; $p=0.045)$ and fast-beta $(25 \mathrm{~Hz} ; p=0.026)$ (Figs. 1,2$)$. Contrasting pre-and post-intervention within the groups, we tested whether post-treatment differences were ascribed to increased fc for the placebo group or decreased connectivity in the Rifaximin group; however, no significant differences were found.

\section{Effects of rifaximin on functional connectivity during CBG}

Contrasting exclusion and inclusion conditions at the baseline for all subjects, we found no significant effect of stress on connectivity in any of the frequencies. Also, no significant differences between the groups were found at baseline. Functional connectivity changes due to the interventions were computed by subtracting the pretreatment connectivity matrices from the post-treatment ones for each CBG condition and each frequency band and then performing a between-group comparison on the computed matrices. The Rifaximin group displayed a network with higher $\mathrm{fc}$ in the theta band $(7 \mathrm{~Hz} ; p=0.038$ ) (Fig. 3) during the inclusion condition. Within-group differences between exclusion and inclusion conditions post-treatment were also found, underlining higher network $\mathrm{fc}$ in theta during the inclusion condition for the Rifaximin group $(p=0.028)$ and exclusion for the placebo one $(p=0.021)$ (Figs. 4,5$)$.

\section{Discussion}

The present study investigated the effects of 7-days Rifaximin intake $(600 \mathrm{mg} / \mathrm{d})$ on MEG functional connectivity in healthy subjects. A double-blind, placebo-controlled, pre-and post-intervention design was undertaken. Functional connectivity was estimated during rest and during a social-stress-inducing protocol (CBG), using imaginary coherency to quantify the connectivity magnitude between cortical brain regions. The resting-state analysis highlighted higher fc networks in different beta bands $(15 \mathrm{~Hz}$ and $25 \mathrm{~Hz})$ during the post-treatment session for the placebo group. However, the lack of any significant effect in the within-group comparison between pre-and post-treatment sessions does not allow us to derive any conclusion regarding the directionality of the aforementioned effect. It is plausible that the placebo group slightly increased connectivity between the treatment session, while Rifaximin, on the contrary, slightly decreased it, thus resulting in a significant group difference in the follow-up session. No effects of social exclusion induced stress on fc were found, comparing CBG conditions. However, differences in theta $(7 \mathrm{~Hz})$ connectivity could be demonstrated between groups following the treatment. The Rifaximin group displayed a network with higher theta fc during the inclusion condition than the placebo group. Within-group comparisons between CBG conditions confirmed the presence of higher theta fc in the inclusion condition for the Rifaximin group after treatment, while the same increase of $\mathrm{fc}$ in theta, yet in the exclusion condition, was found for the placebo group. Given the presence of the insular cortex in all the identified networks, group differences in functional connectivity might be mediated by this area in case of alterations of gut microbiota following Rifaximin intake. However, considering the study's novelty and the current lack of wellfounded theories on the effects of antibiotics on functional connectivity, clear interpretations of the results are 


\section{$15 \mathrm{~Hz}$ plc $>$ rfx}

A
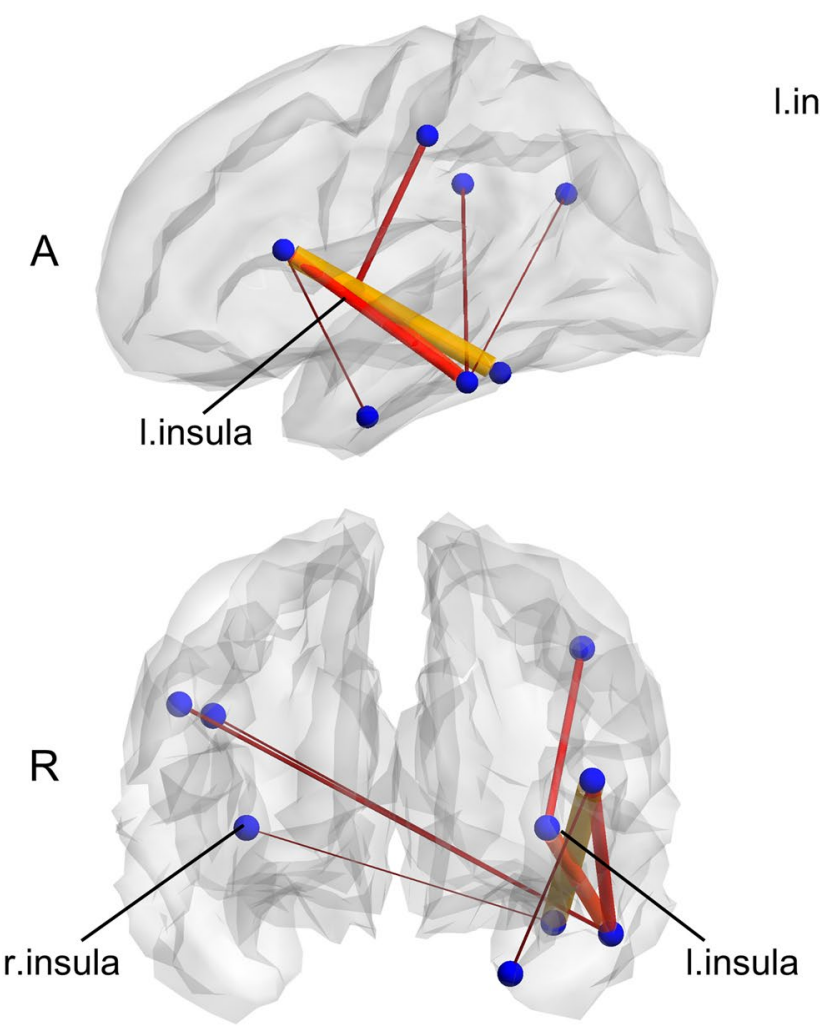

A

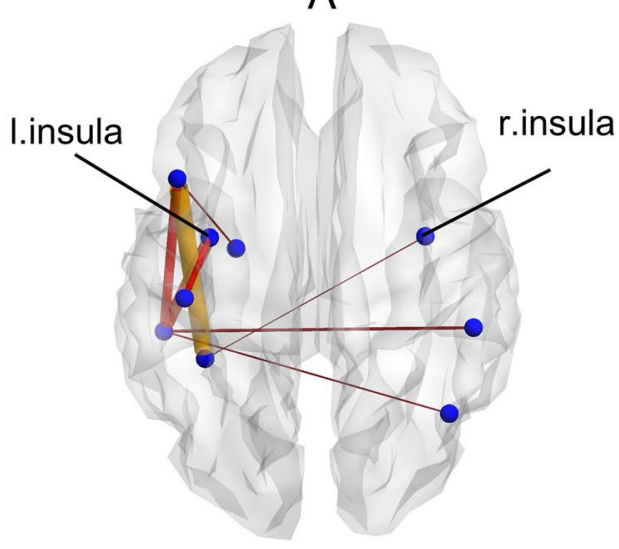

$\mathrm{t}$

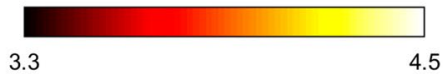

Figure 1. Between groups functional connectivity comparison in post-treatment session. The placebo group displays two networks with increased connectivity in slow and fast beta band $(15 \mathrm{~Hz})$ compared to the Rifaximin group. Clusters of higher connectivity are located mainly in the left hemisphere, involving frontal, temporal, and parietal areas. To note the presence of the insular cortex within the networks. Color and size of the edges are proportional to t-statistic $(\mathrm{t})$ value ( $A$ anterior, $R$ right).

difficult and, therefore, predominantly of speculative nature. Furthermore, while the involvement of the insular cortex was consistent across both the resting state and the CBG protocol, frequencies of coherence-sensitive to tasks, contexts, and brain states-differed between paradigms.

Coherence here considered as a measure of functional connectivity ${ }^{67}$ has been proposed as a mechanism allowing flexible communication between groups of neurons ${ }^{68}$, subserving the integration of multisensory information and thus allowing for multisensory perception ${ }^{69}$, as well as for our cognitive flexibility ${ }^{70}$. In our study, group differences of coherence levels in beta bands were found after treatment. Coupling in beta-band activity has been proposed to be associated with the maintenance of the status quo, resulting in a strong inhibition of behavioral and cognitive changes during a pathological exacerbation of beta-band coherence ${ }^{71}$. Here, we speculate that lower connectivity found in beta for the Rifaximin group during resting state might indicate an enhancement of cognitive flexibility following GM-antibiotic-alterations. One might argue that the lack of any significance within the effect of treatment for Rifaximin group does not support this interpretation. However, since both the group were exposed to the same variables in terms of time, measurement repetition, and intervention paradigm, it is justified to assume that lower beta networks coherence found in Rifaximin group is due to the active intervention.

Cognitive flexibility is defined as the ability to shift between "cognitive status," adapting cognitive processing strategies to changes in the environment ${ }^{72}$. Low cognitive flexibility has been associated with greater negative appraisal of stressful situations ${ }^{73}$. Clinically, impairment in cognitive flexibility has been linked to excessive rumination in depression ${ }^{74}$, emotion dysregulation in mood and anxiety ${ }^{75}$, obsessive-compulsive ${ }^{76}$, and eating disorders ${ }^{77}$. Potential evidence supporting the speculation of a GM-cognitive flexibility link are offered by the irritable bowel syndrome. IBS is characterized by a broader group of symptoms that include abdominal pain, diarrhea, and/or constipation ${ }^{78}$, which have been linked with alterations in GM composition ${ }^{79-86}$. This condition often coexists with different psychological disorders ${ }^{87-89}$. Comorbidity between IBS and depression, a mental disorder that is characterized by a strong focus on negative expectations and impaired cognitive flexibility ${ }^{73,90-92}$, is documented ${ }^{93-95}$. Furthermore, increased perseverative error in the Wisconsin Card Sorting Test $^{96}$ has been observed in IBS patient ${ }^{97,98}$, together with altered brain activity in prefrontal areas, insular cortex, and hippocampus ${ }^{97}$. These findings might indicate impairments in cognitive flexibility, thus corroborating the speculation of a possible connection between GM and high cognitive functions, such as mental flexibility.

Regarding the condition-related enhancement in theta coherence, we speculate state-dependent processing of social stress, which is different following placebo or Rifaximin intake. Theta oscillations have been associated 


\section{$25 \mathrm{~Hz}$ plc>rfx}

A
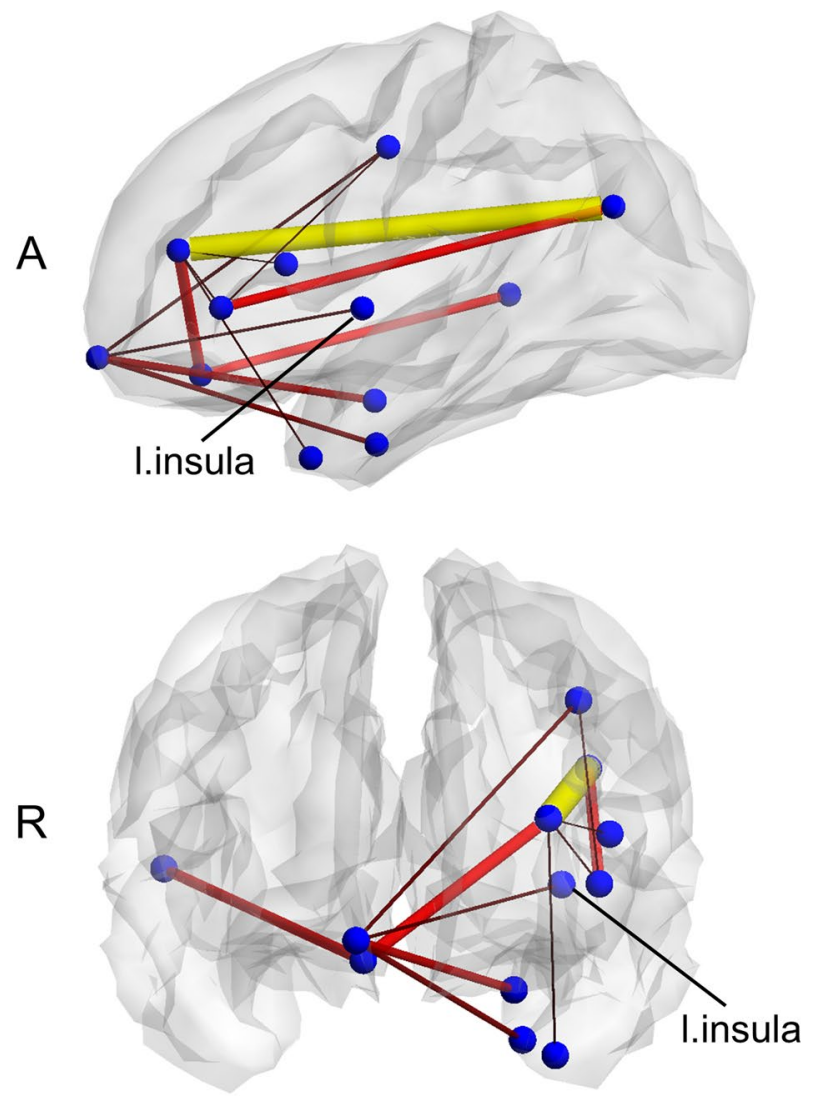

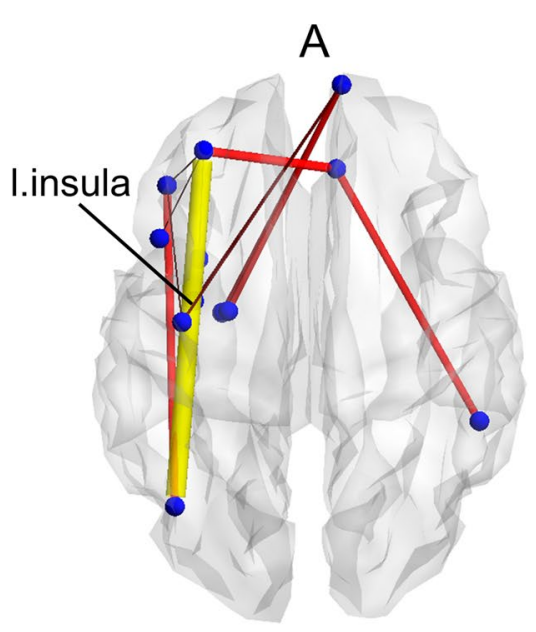

$\mathrm{t}$

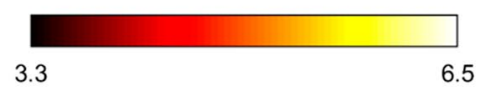

Figure 2. Between groups functional connectivity comparison in post-treatment session. The placebo group displays two networks with increased connectivity in slow and fast beta band $(25 \mathrm{~Hz})$ compared to the Rifaximin group. Clusters of higher connectivity are located mainly in the left hemisphere, involving frontal, temporal, and parietal areas. To note the presence of the insular cortex within the networks. Color and size of the edges are proportional to $\mathrm{t}$-statistic $(\mathrm{t})$ value ( $A$ anterior, $R$ right).

with memory processes ${ }^{99}$. In particular, enhanced theta coherence has been repeatedly associated with successful memory encoding and retrieval ${ }^{100-107}$. Since stress exposure has been seen to enhance emotional aspects of episodic memory ${ }^{108-110}$, our condition-dependent theta coherence network enhancement might reflect a bias towards negative (placebo) and positive (Rifaximin) aspects during the consolidation of the experience of stressful situations. In one study ${ }^{111}$, using word-related memory tests, IBS patients displayed a significant bias towards emotionally negative words, the same as displayed in depressed patients. This study highlights the presence of a confirmatory bias for negative material processing in a condition of GM alteration, again suggesting a possible connection between gut microbiota composition, and an alteration in memory function, that might reasonably be reflected by a condition-dependent enhancement of coherence networks. Noteworthy, the improvement of emotional well-being following the intake of Rifaximin reported in our previous study ${ }^{64}$ is well consistent with here proposed interpretation of Rifaximin-induced brain network effects indicating a positive bias during the consolidation of emotional experiences.

Finally, our hypothesis concerning the presence of the insula, given its important role in interoception ${ }^{65}$, has been confirmed in all the highlighted networks, oscillating either in beta or theta frequency. Therefore, we propose the insular cortex to be a critical node in mediating changes in fc following GM alteration. Several mechanisms have been suggested through which GM alterations might affect CNS and cognitive functions, involving neuro-vagal, endocrine, and immune pathways ${ }^{3,5,6,9}$. The insular cortex is known to be the converging point of the spinothalamocortical pathway, which processes and integrates interoceptive information, transmitted primarily through vagal afferents ${ }^{65}$. These observations highlight the relevance of the insula within the microbiota-gut-brain axis and support the hypothesis of an insula-mediated fc alteration following changes in GM composition. In support of our hypothesis, previous MRI studies described structural and functional alteration of the insula in IBS patients ${ }^{112-116}$. One study ${ }^{117}$ reported significantly increased cortical thickness of the right posterior insula in a group of diarrhea-predominant IBS female patients supposedly related with enhanced interoceptive monitoring. Resting-state insula seed-based analysis in female IBS patients ${ }^{118}$ displayed negative fc between dorsal anterior insula, medial prefrontal cortex, and precuneus, which are a key node of the default mode network $(\mathrm{DMN})$, suggesting the role of the insula in modulating intrinsic fc of major networks and in the 


\section{$7 \mathrm{~Hz} \Delta \mathrm{rfx}$ incl $>\Delta \mathrm{plc}$ _incl}
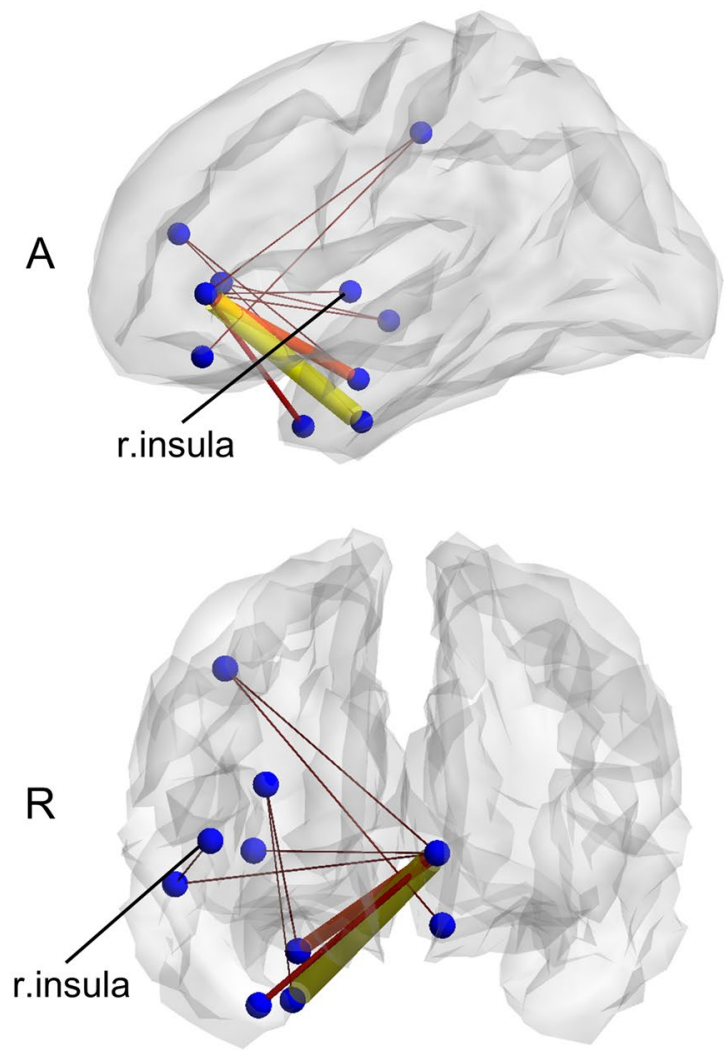

A

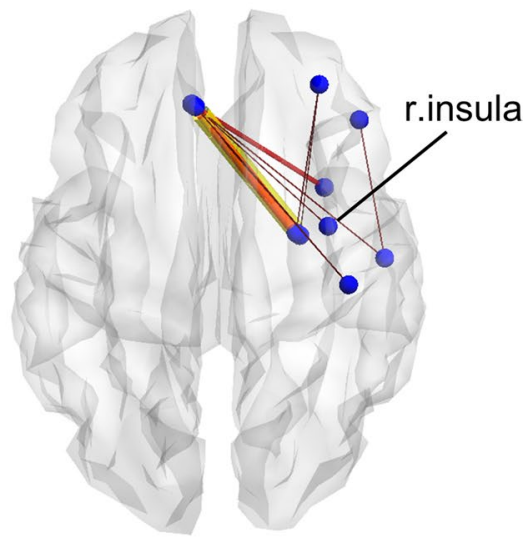

$\mathrm{t}$

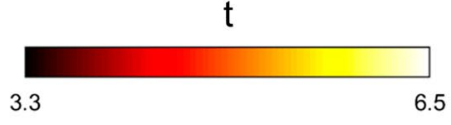

Figure 3. Between groups functional connectivity comparison during CBG in post-treatment session. Theta $(7 \mathrm{~Hz})$ higher $\mathrm{fc}$ connectivity network found in Rifaximin group during the inclusion condition. The cluster of higher connectivity involves the frontal and temporal lobe, mainly in the right hemisphere. The right insular cortex is highlighted. Size and color of the edges are proportional to the t-statistic $(\mathrm{t})$ value $(\Delta=$ post-pre matrices, $A$ anterior, $R$ right).

pathophysiology of IBS. Relevantly, a recent study on tobacco smokers ${ }^{119}$ using seed-based insula analysis at rest showed a middle insular resting-state fc to frontal and cerebellar areas associated with GM structure, bacterial diversity, and genera (Prevotella and Bacteroides).

With respect to the effects of Rifaximin on human GM and CNS, so far, evidence emerges mainly from studies on pathological conditions. Reasonably, this fact entails strong limitations on the derivation of any conclusion about a causative role of the treatment since any observed outcome might result from an amelioration of the pathological condition, rather than a consequence of a GM-Rifaximin-induced alteration on CNS. Nevertheless, studying the effects of Rifaximin in patients certainly offers an interesting starting point. As yet, Rifaximin has been successfully used in treating IBS $^{120-124}$, and proved to have the greatest effect on IBS symptoms among different other antibiotics ${ }^{125}$. Interestingly, however, in cirrhotic patients with minimal hepatic encephalopathy (MHE), a condition without obvious clinical manifestation characterized by cognitive impairment in attention, vigilance, and integrative function ${ }^{126}$, Rifaximin has been seen to improve cognitive functioning and health-related quality of life ${ }^{127-131}$. To note that in MHE, which has been linked with significant alterations in the gut microbiome associated with cognition ${ }^{132}$, among the different improved cognitive functions, cognitive flexibility ${ }^{127}$ and working memory ${ }^{131}$ have been reported to improve after Rifaximin treatment.

A multifactorial explanation has been proposed to clarify the nature of the aforementioned improvements, highlighting the potential mechanisms through which Rifaximin might modulate CNS functioning. Reduced endotoxemia and altered microbiota-associated metabolic function, with a change in fatty acids production, have been reported following Rifaximin treatment ${ }^{128}$. Furthermore, it seems that the mechanism of action of Rifaximin, despite its role as a gastrointestinal-targeting antibiotic, might extend beyond the GM, modulating the production of inflammatory cytokines and intestinal permeability ${ }^{133}$. Finally, Rifaximin treatments have been seen to promote beneficial gut bacteria such as Bifidobacteria and Lactobacilli in patients with gastrointestinal and liver disorders ${ }^{134,135}$. Based on these observations, it is likely that Rifaximin intake might also alter brain functional connectivity in healthy subjects through its focused and yet broad actions on GM and the gastrointestinal tract.

Although the results displayed the potential effects of Rifaximin-induced GM modulation on CNS connectivity, the study presents several limitations. First, in reason of the pilot nature of the study conceived to explore the feasibility of the approach used to generate the hypothesis to be tested in further experiments, only 16 participants 


\section{$7 \mathrm{~Hz} \Delta$ plc_excl> $\Delta$ plc_incl}

A
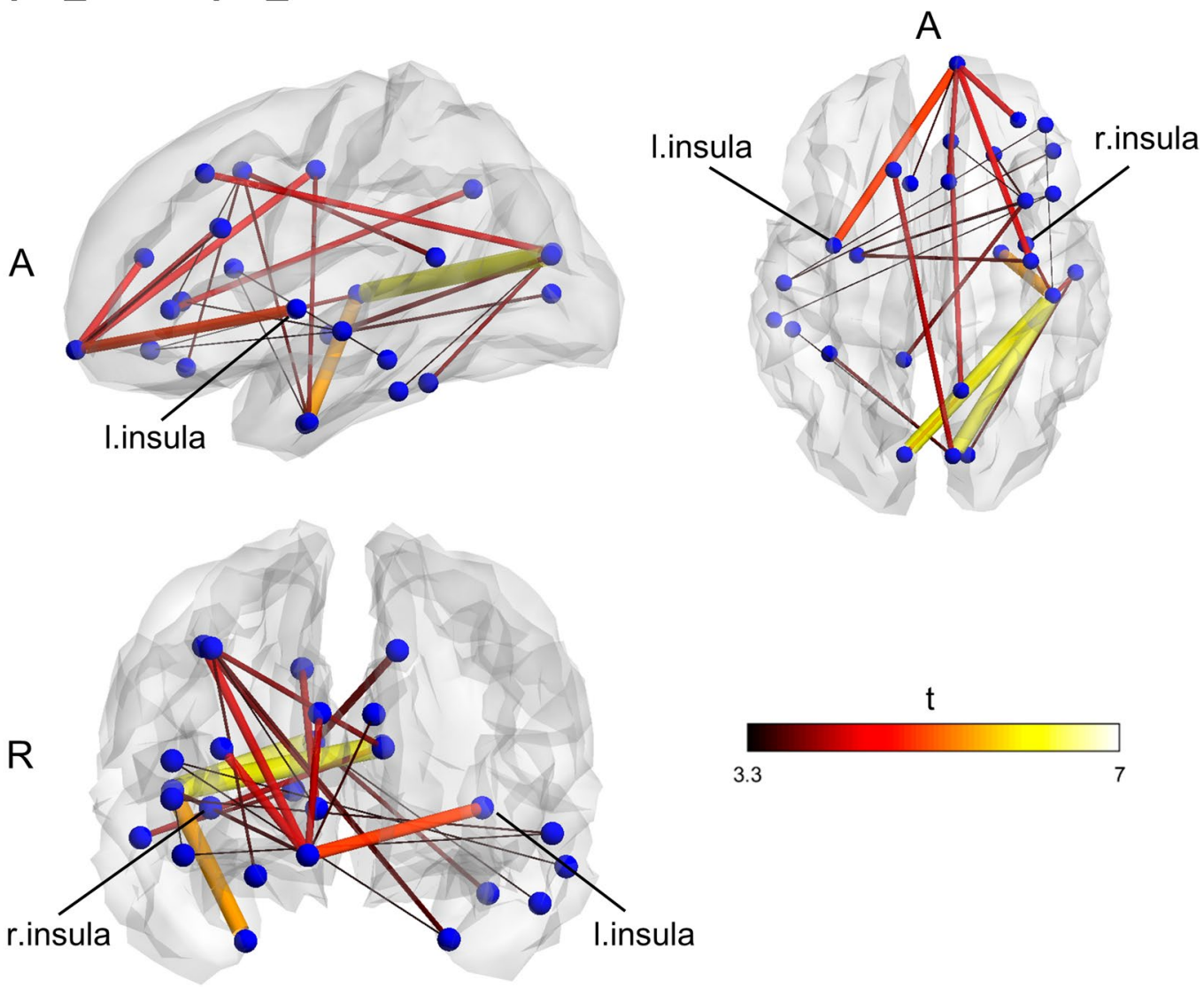

Figure 4. Within groups, functional connectivity comparison between exclusion and inclusion condition in post-treatment session. Placebo group $(\Delta$ plc) displays a higher functional connectivity network in theta during the exclusion condition of the CBG as compared to the inclusion one. The identified cluster widely extent across all the brain, involving frontal, parietal, temporal, and occipital areas. To note the presence of the insula again, size and color of the edges are proportional to the t-statistic ( $\mathrm{t})$ value ( $\Delta=$ post-pre matrices, $A$ anterior, $R$ right).

were recruited. Therefore, the here presented preliminary results need to be replicated and validated. In addition, the absence of significant differences in age between the groups is most likely due to the large variability of the participants' ages rather than to a close match of the groups. In future studies, it is highly recommended to better control for age and other factors such as personality traits to increase the specificity of the interpretation of the results. Second, unlike previous investigations using CBG as a social-stressor protocol ${ }^{136,137}$, no evidence signaling the involvement of different networks for exclusion and inclusion conditions was found in the baseline. The reason for the difference is unclear and entails some limitations for the interpretation of the results in the post-treatment session. It is possible that during the baseline session, all the participants were under the same level of stress/excitation regardless of the condition due to the unfamiliarity with the experimental situation, resulting in a flattening of any significant difference in $\mathrm{fc}$. In a future study, it might be reasonable to introduce a task familiarization session before the beginning of the experiment in order to avoid an unfamiliarity effect that could overwrite any Rifaximin and GM-induced fc difference. Nonetheless, this was the first study investigating the impact of social stress on MEG fc, making it difficult to compare our results with previously published work using $\mathrm{fc} \mathrm{MRI}{ }^{136,137}$. Finally, again in reason of the exploratory nature of the study, no stool samples for microbiome analysis and additional peripheral physiological parameters had been collected. The lack of stool sampling, to include in further research, prevent any clearer conclusion about GM alterations that could have helped to shed light on the mechanism of action through which Rifaximin might affect brain fc. Regarding the physiological parameters, the stress effect of the CBG and the health status of the participant have here been investigated only through self-reported questionnaires ${ }^{64}$. In further investigation, physiological and/or hormonal responses should be considered to increase the objectivity of the results.

In conclusion, our findings showed widespread functional connectivity alterations during rest and during a stress paradigm, following 7-days Rifaximin intake. The presence of the insular cortex in all the highlighted clusters might indicate its potential role in mediating changes in $\mathrm{fc}$, signaling commensal microbiota alterations. Connectivity in beta and theta frequencies was affected, suggesting possible relations with higher cognitive function, such as cognitive flexibility and memory encoding. Further studies are necessary to replicate the results and confirm these hypotheses. A cognitive task-based multimodal neuroimaging study investigating precisely flexibility and memory functioning should be addressed. A larger sample and the inclusion of other test groups 


\section{$7 \mathrm{~Hz} \Delta \mathrm{rfx} \_$incl $>\Delta \mathrm{rfx} \_$excl}
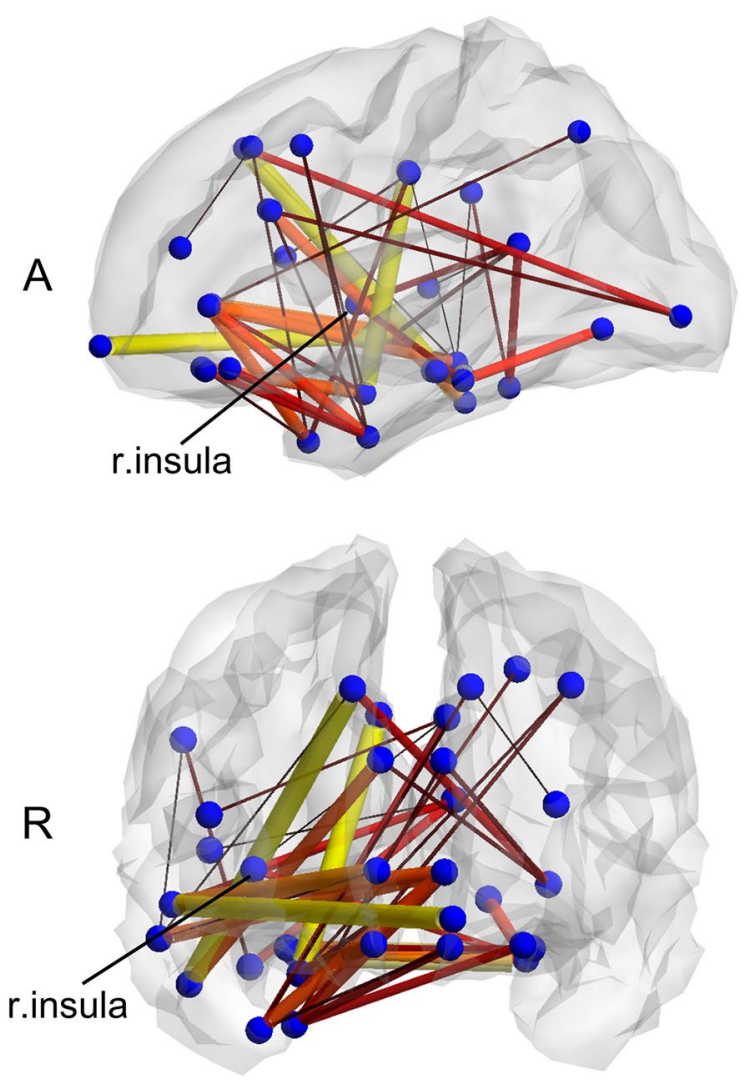
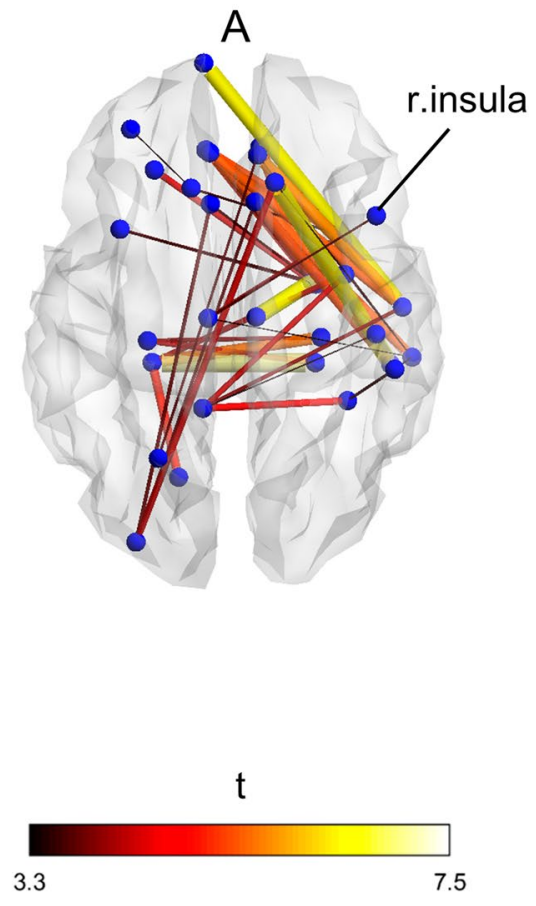

Figure 5. Within groups, functional connectivity comparison between exclusion and inclusion condition in post-treatment session. An opposite pattern was found for the Rifaximin group $(\Delta \mathrm{rfx})$, displaying a higher functional connectivity network in theta during the inclusion condition. Again, the network is largely widespread across the brain. To note the presence of the insula again, size and color of the edges are proportional to the $\mathrm{t}$-statistic $(\mathrm{t})$ value ( $\Delta=$ post-pre matrices, $A$ anterior, $R$ right).

(e. g., probiotic, and IBS patients) might offer a more global picture of the mechanism through which GM affects the CNS.

\section{Methods}

Subjects and study design. Sixteen healthy participants were recruited for the study ( 9 males; mean age: $27.00 \pm 6.39$ SD, range 22-49 years of age; BMI: $22.21 \pm 1,94$ SD). Eight participants completed the intervention with Rifaximin (rfx, 6 males, mean age: $26.50 \pm 2.98$ SD, range $24-31$ years of age; BMI: $22.48 \pm 1.64$ SD) and eight with placebo (plc, 3 males, mean age: $27.50 \pm 8.83$ SD, range $22-49$ years of age; BMI: $21.94 \pm 2.28 \mathrm{SD}$ ). Age and BMI were not significantly different between placebo and Rifaximin groups. Exclusion criteria were MEG/ MR incompatibility, use of antibiotics within the last 2 months, and psychiatric/gastrointestinal disorder. All participants signed informed consent before joining the study. The protocol has been approved by the Ethics Board of the University of Tübingen Medical School (No. 503/2015BO1, approved on 26.08.2015).

A randomized, double-blinded, placebo-controlled design with pre-and post-intervention assessment was used. All participants underwent two MEG recording sessions, before (baseline- $\mathrm{t} 0$ ) and after 7 days of treatment (t1) either with Rifaximin $(3 \times 200 \mathrm{mg} / \mathrm{d})$ or placebo pills. During all experimental periods, subjects had to avoid the consumption of probiotic and prebiotic_containing foods. Both groups were tested during rest and while playing a cyber ball game ${ }^{138}$. CBG is an online ball-tossing game in which participants believe that they are playing with two real "others." In contrast, the dynamics are actually controlled by the experimenter, which can manipulate the inclusion rate of the participant in the game, eliciting interpersonal ostracism, and social stress. In our experiment, the paradigm consists of 4 game sessions, programmed to vary inclusion (incl) and exclusion (excl) conditions. During the inclusion block, each of the players had an equal chance to receive the ball, while during the exclusion block, the "real" participant was largely excluded from the game. The $1 / 3$ of the total 108 trials of the inclusion block, when the participant just observed the virtual players were throwing the ball to each other, were called "not my turns" events. These 36 "not my turn" trials were then compared to the 36 so-called "rejection" trials during the exclusion condition. The beginning of the trial was defined by randomly 
presenting the ball for 500 to $2000 \mathrm{~ms}$. After one of the players threw the ball, this was moving for $2000 \mathrm{~ms}$ before reaching the target player.

Data recording. MEG data were recorded at the MEG-Center of the Universitätsklinikum Tübingen using a whole-head 275 channels MEG system (CTF Inc., Vancouver, Canada) located in a magnetically shielded room. MEG signals were sampled at $585.94 \mathrm{~Hz}$, with an anti-aliasing filter set at $146.24 \mathrm{~Hz}$. First, 5 min of spontaneous resting-state activity was recorded. Participants were measured in a sitting position and were instructed to move as little as possible, keeping their eyes closed and not to fall asleep. Following the rs session, the CBG paradigm was initiated. Task instructions were projected onto a screen in front of the subject. Participants had to fixate the screen and interact with the game by pressing two different buttons (left and right) of a response box, depending on the direction in which they wanted to throw the virtual ball.

4.3 For the reconstruction of neuromagnetic sources, head anatomical data were acquired using a Siemens Magnetom Trio or Prisma 3 T scanner (Siemens AG, Erlangen, Germany) with a 12-channel head coil. Highresolution ( $1 \mathrm{~mm}$, isotropic voxels) T1-weighted whole-head structural images were acquired for each participant on different days than the MEG recording. To further coregister the two datasets, we first localized the position of the head during the MEG recording using three fiducial coils (left/right preauricular points and nasion), and we monitored the head motion throughout the experiment with a threshold limit of $5 \mathrm{~mm}$. At the time of the MR scan, we used MRI-visible fiducials, located at the same position as the head coils, to make it easier to localize the correct points further when we realigned the structural images according to the CTF coordinate space of the MEG system.

Data analysis. MEG data were analyzed using MATLAB (MathWorks Inc., Natick, Massachusetts USA), NBS Connectome ${ }^{139}$, and FieldTrip, an open-source MATLAB toolbox specifically developed for electrophysiological data analysis ${ }^{140}$. BrainNet Viewer ${ }^{141}$ was used for the visualization of the results. Head anatomical data, from MRI acquisition, were processed using Fieldtrip, the software package FreeSurfer ${ }^{142-144}$ and AFNI/ SUMA $^{145-147}$.

Continuously recorded data were first filtered to reduce the $50 \mathrm{~Hz}$ power line noise using the DFT filter function provided on fieldtrip, which applies a notch filter to the data. Resting-state data were segmented into 150 segments of $2000 \mathrm{~ms}$ length. CBG data were segmented in intervals ranging from $1000 \mathrm{~ms}$ pre- and $2000 \mathrm{~ms}$ post-stimulus onset (triggered by the action of throwing the ball by one of the players) and baseline corrected. Because the CBG paradigm in our experiment alternated inclusion and exclusion conditions in a four games session, we appended all the trials belonging to the same condition. Trials in which the other players threw the ball towards each other during the inclusion blocks were labeled as "inclusion," and those during the exclusion blocks as "exclusion." A total of 72 trials for each condition were defined. Next, we manually excluded any trial whose variance exceeded $10^{25} \mathrm{~T}^{2}$ in any channel using the rejectvisual function provided in FieldTrip. In addition, an independent component analysis to remove non-cortical physiological activity (eye-blink, heartbeat, and muscular components) was performed.

For spectrum decomposition and source analysis, we first selected different frequencies of interest, ranging from 5 to $29 \mathrm{~Hz}$, in steps of $2 \mathrm{~Hz}$, with a smoothing window of $\pm 1 \mathrm{~Hz}$. Then a common spatial filter was computed (independently for resting state and CBG) using partial canonical correlation/coherence (PCC) as a beamformer method $^{148}$. Subsequently, the common filter was applied separately to pre/post and inclusion/exclusion conditions for source reconstruction. This "common filter" approach was used to minimize the possibility of introducing a bias towards one condition during the source estimation, related to differences between the filters themselves rather than an actual difference between conditions. A cortical sheet serving as a source model was used for source reconstruction. In consideration of MEG's sensitivity mostly to tangential and cortical sources ${ }^{149,150}$, cerebellum and subcortical regions (except bilateral hippocampus and amygdala) were not included in the analysis. All the steps of the pipeline are illustrated in Fig. 6.

In order to co-register the neuromagnetic activities acquired with MEG to anatomical structures, the topology of individual cortices was extracted from individual MRI scans as a three-dimensional mesh. After preprocessing and spectral decomposition, source reconstruction was performed using a cortical sheet with 2004 nodes as source model. Absolute imaginary coherency was used to quantify functional connectivity between nodes of the cortical mesh. Finally, a parcellation scheme was applied to reduce data dimensionality before the statistical analysis.

Creation of the cortical source model. T1 weighted anatomical images were first realigned according to MNI's RAS coordinate system and then resliced, defining $1 \mathrm{~mm}$ thickness for the slice and specifying $256 \times 256 \times 256 \mathrm{~mm}^{3}$ volume. In addition, a skull stripping of the anatomical image was performed to separate the brain from non-brain tissue using FreeSurfer. We visually inspected the results, manually corrected any error due to too aggressive/conservative skull-stripping, and then proceeded with the automatic segmentation function provided by the software. Each hemisphere was warped into a topological sphere (with white/gray matter boundary defined from FreeSurfer automatic segmentation). Cortex-based inter-subject realignment was done on the warped spheres ${ }^{143,144}$. To decimate and interpolate the vertices generated by FreeSurfer $(>100,000)$ to 1002 common vertices for the hemisphere, SUMA (Surface Mapper from AFNI suite) was used. This procedure was done to increase source data handling and allow group-level statistical analyses. The same method was

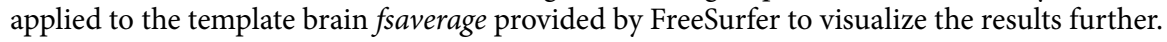

Network analysis. NBS connectome ${ }^{139}$, implementing the nonparametric network-based statistic (NBS) method, was used for the network analysis. The nonparametric way of testing was chosen due to the non-nor- 


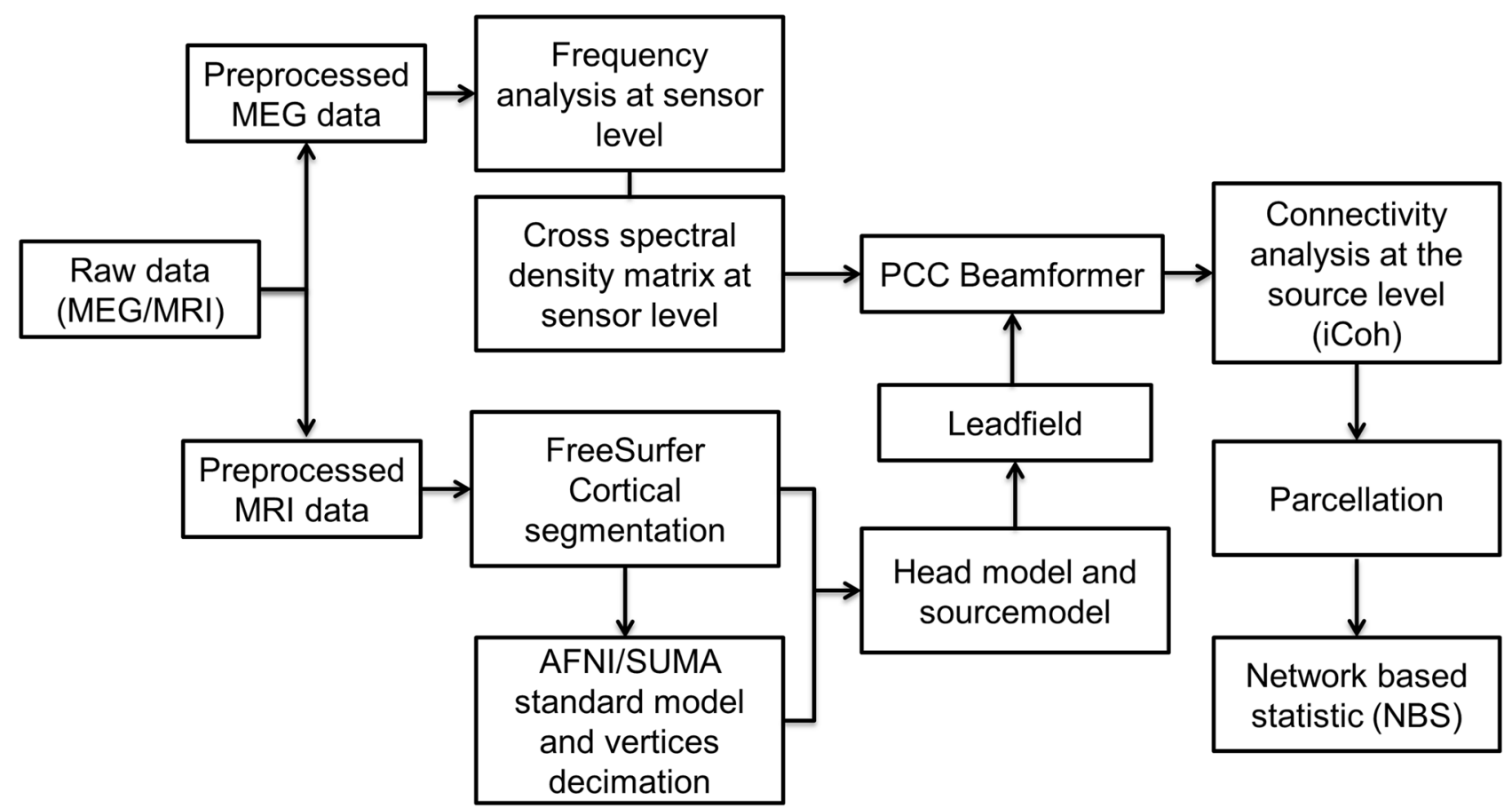

Figure 6. Data analysis pipeline.

mally distributed values of coherence. Parcellated connectivity matrices were used as input for the analysis. The statistical model was defined in terms of general linear model (GLM), and we chose a test statistic threshold of $3.3(\mathrm{t})$. All between and within-group comparisons used either independent or paired sample $t$ test, respectively, as a first step of the cluster identification. All tests were corrected for multiple comparisons.

The NBS method involved four different steps. First, it runs a massive univariate test to define a test statistic value for each connection. Second, it compares the t-statistic value of each connection, with the previously defined threshold, identifying all the supra-threshold connections. The next step determines the presence of any topological clusters among the sets of supra-threshold connections. Finally, it permutes the data, repeating the first three steps $n$ times (5000 permutations). Data are randomly relabeled depending on the test to be accomplished, involving either the two treatment groups (Rifaximin and placebo), the sessions (pre-and posttreatment), or the CBG conditions (inclusion and exclusion). The size of the largest cluster is recorded after each permutation. Based on all permutations, an empirical null distribution for the size of the largest cluster was generated. The error probability of the observed cluster difference's error probability was expressed in a one-sided FWER-corrected $p$ value ${ }^{151}$ was based on the null distribution.

Received: 15 June 2020; Accepted: 18 January 2021

Published online: 24 February 2021

\section{References}

1. Rhee, S. H., Pothoulakis, C. \& Mayer, E. A. Principles and clinical implications of the brain-gut-enteric microbiota axis. Nat. Rev. Gastroenterol. Hepatol. 6, 306-314 (2009).

2. Grenham, S., Clarke, G., Cryan, J. F. \& Dinan, T. G. Brain-Gut-Microbe Communication in Health and Disease. Front. Physiol. 2, $24(2011)$.

3. Cryan, J. F. \& Dinan, T. G. Mind-altering microorganisms: the impact of the gut microbiota on brain and behaviour. Nat. Rev. Neurosci. 13, 701-712 (2012).

4. Mayer, E. A., Knight, R., Mazmanian, S. K., Cryan, J. F. \& Tillisch, K. Gut Microbes and the Brain: paradigm shift in neuroscience. J. Neurosci. 34, 15490-15496 (2014).

5. Mayer, E. A., Tillisch, K. \& Gupta, A. Gut/brain axis and the microbiota. J. Clin. Investig. 125, 926-938 (2015).

6. Sharon, G., Sampson, T. R., Geschwind, D. H. \& Mazmanian, S. K. The central nervous system and the gut microbiome. Cell 167, 915-932 (2016).

7. Yarandi, S. S., Peterson, D. A., Treisman, G. J., Moran, T. H. \& Pasricha, P. J. Modulatory effects of gut microbiota on the central nervous system: how gut could play a role in neuropsychiatric health and diseases. J. Neurogastroenterol. Motil. 22, 201-212 (2016).

8. Martin, C. R., Osadchiy, V., Kalani, A. \& Mayer, E. A. The brain-gut-microbiome axis. Cell. Mol. Gastroenterol. Hepatol. 6, 133-148 (2018).

9. Ma, Q. et al. Impact of microbiota on central nervous system and neurological diseases: the gut-brain axis. J. Neuroinflamm. 16, 53 (2019).

10. Heijtz, R. D. et al. Normal gut microbiota modulates brain development and behavior. Proc. Natl. Acad. Sci. 108, 3047-3052 (2011).

11. Sudo, N. et al. Postnatal microbial colonization programs the hypothalamic-pituitary-adrenal system for stress response in mice. J. Physiol. 558, 263-275 (2004). 
12. Bravo, J. A. et al. Ingestion of lactobacillus strain regulates emotional behavior and central GABA receptor expression in a mouse via the vagus nerve. Proc. Natl. Acad. Sci. 108, 16050-16055 (2011).

13. Savignac, H. M., Kiely, B., Dinan, T. G. \& Cryan, J. F. Bifidobacteria exert strain-specific effects on stress-related behavior and physiology in BALB/c mice. Neurogastroenterol. Motil. 26, 1615-1627 (2014).

14. Ait-Belgnaoui, A. et al. Prevention of gut leakiness by a probiotic treatment leads to attenuated HPA response to an acute psychological stress in rats. Psychoneuroendocrinology 37, 1885-1895 (2012).

15. Ait-Belgnaoui, A. et al. Bifidobacterium longum and lactobacillus helveticus synergistically suppress stress-related visceral hypersensitivity through hypothalamic-pituitary-adrenal axis modulation. J. Neurogastroenterol. Motil. 24, 138-146 (2018).

16. Jang, S.-E. et al. Gastrointestinal inflammation by gut microbiota disturbance induces memory impairment in mice. Mucosal Immunol. 11, 369-379 (2018).

17. Lu, J. et al. Microbiota influence the development of the brain and behaviors in C57BL/6J mice. PLoS ONE 13, e0201829 (2018).

18. Zhan, G. et al. Abnormal gut microbiota composition contributes to cognitive dysfunction in SAMP8 mice. Aging 10, 1257-1267 (2018).

19. Sanguinetti, E. et al. Microbiota signatures relating to reduced memory and exploratory behaviour in the offspring of overweight mothers in a murine model. Sci. Rep. 9, 12609 (2019).

20. Ericsson, A. C. \& Franklin, C. L. Manipulating the gut microbiota: methods and challenges. ILAR J. 56, 205-217 (2015).

21. Wu, G. D. et al. Linking long-term dietary patterns with gut microbial enterotypes. Science 334, 105-108 (2011).

22. Claesson, M. J. et al. Gut microbiota composition correlates with diet and health in the elderly. Nature 488, 178-184 (2012).

23. Konturek, P. C., Brzozowski, T. \& Konturek, S. J. Stress and the gut: pathophysiology, clinical consequences, diagnostic approach and treatment options. J. Physiol. Pharmacol. Off. J. Pol. Physiol. Soc. 62, 591-599 (2011).

24. Queipo-Ortuño, M. I. et al. Influence of red wine polyphenols and ethanol on the gut microbiota ecology and biochemical biomarkers. Am. J. Clin. Nutr. 95, 1323-1334 (2012).

25. Biedermann, L. et al. Smoking cessation induces profound changes in the composition of the intestinal microbiota in humans. PLoS ONE 8, e59260 (2013).

26. Jia, W., Li, H., Zhao, L. \& Nicholson, J. K. Gut microbiota: a potential new territory for drug targeting. Nat. Rev. Drug Discov. 7, 123-129 (2008).

27. Preidis, G. A. \& Versalovic, J. Targeting the human microbiome with antibiotics, probiotics, and prebiotics: gastroenterology enters the metagenomics era. Gastroenterology 136, 2015-2031 (2009).

28. Cani, P. D. \& Delzenne, N. M. The gut microbiome as therapeutic target. Pharmacol. Ther. 130, 202-212 (2011).

29. Logan, A. C. \& Katzman, M. Major depressive disorder: probiotics may be an adjuvant therapy. Med. Hypotheses 64, 533-538 (2005).

30. Dinan, T. G., Stanton, C. \& Cryan, J. F. Psychobiotics: a novel class of psychotropic. Biol. Psychiatry 74, 720-726 (2013).

31. Wall, R. et al. Bacterial neuroactive compounds produced by psychobiotics. In Microbial Endocrinology: The Microbiota-Gut-Brain Axis in Health and Disease (eds Lyte, M. \& Cryan, J. F.) 221-239 (Springer, Berlin, 2014). https://doi.org/10.1007/978-1-49390897-4_10.

32. Sarkar, A. et al. Psychobiotics and the manipulation of bacteria-gut-brain signals. Trends Neurosci. 39, 763-781 (2016).

33. Wang, H., Lee, I.-S., Braun, C. \& Enck, P. Effect of probiotics on central nervous system functions in animals and humans: a systematic review. J. Neurogastroenterol. Motil. 22, 589-605 (2016).

34. Bambury, A., Sandhu, K., Cryan, J. F. \& Dinan, T. G. Finding the needle in the haystack: systematic identification of psychobiotics. Br. J. Pharmacol. 175, 4430-4438 (2018).

35. Bermúdez-Humarán, L. G. et al. From probiotics to psychobiotics: live beneficial bacteria which act on the brain-gut axis. Nutrients 11, 890 (2019).

36. Benton, D., Williams, C. \& Brown, A. Impact of consuming a milk drink containing a probiotic on mood and cognition. Eur. J. Clin. Nutr. 61, 355-361 (2007).

37. Messaoudi, M. et al. Beneficial psychological effects of a probiotic formulation (Lactobacillus helveticus R0052 and Bifidobacterium longum R0175) in healthy human volunteers. Gut Microbes 2, 256-261 (2011).

38. Steenbergen, L., Sellaro, R., van Hemert, S., Bosch, J. A. \& Colzato, L. S. A randomized controlled trial to test the effect of multispecies probiotics on cognitive reactivity to sad mood. Brain. Behav. Immun. 48, 258-264 (2015).

39. Takada, M. et al. Probiotic Lactobacillus casei strain Shirota relieves stress-associated symptoms by modulating the gut-brain interaction in human and animal models. Neurogastroenterol. Motil. 28, 1027-1036 (2016).

40. Tillisch, K. et al. Consumption of fermented milk product with probiotic modulates brain activity. Gastroenterology 144, 13941401.e4 (2013).

41. Pinto-Sanchez, M. I. et al. Probiotic bifidobacterium longum NCC3001 reduces depression scores and alters brain activity: a pilot study in patients with irritable bowel syndrome. Gastroenterology 153, 448-459.e8 (2017).

42. Bagga, D. et al. Probiotics drive gut microbiome triggering emotional brain signatures. Gut Microbes 9, 486-496 (2018).

43. Bagga, D. et al. Influence of 4-week multi-strain probiotic administration on resting-state functional connectivity in healthy volunteers. Eur. J. Nutr. 58, 1821-1827 (2019).

44. Allen, A. P. et al. Bifidobacterium longum 1714 as a translational psychobiotic: modulation of stress, electrophysiology and neurocognition in healthy volunteers. Transl. Psychiatry 6, e939 (2016).

45. Wang, H., Braun, C., Murphy, E. F. \& Enck, P. Bifidobacterium longum $1714^{\mathrm{TM}}$ strain modulates brain activity of healthy volunteers during social stress. Am. J. Gastroenterol. 114, 1152-1162 (2019).

46. Kelly, J. R. et al. Lost in translation? The potential psychobiotic Lactobacillus rhamnosus (JB-1) fails to modulate stress or cognitive performance in healthy male subjects. Brain. Behav. Immun. 61, 50-59 (2017).

47. Jernberg, C., Löfmark, S., Edlund, C. \& Jansson, J. K. Long-term ecological impacts of antibiotic administration on the human intestinal microbiota. ISME J. 1, 56-66 (2007).

48. Buffie, C. G. et al. Profound alterations of intestinal microbiota following a single dose of clindamycin results in sustained susceptibility to Clostridium difficile-induced colitis. Infect. Immun. 80, 62-73 (2012).

49. Modi, S. R., Collins, J. J. \& Relman, D. A. Antibiotics and the gut microbiota. J. Clin. Investig. 124, 4212-4218 (2014).

50. Desbonnet, L. et al. Gut microbiota depletion from early adolescence in mice: implications for brain and behaviour. Brain. Behav. Immun. 48, 165-173 (2015).

51. Fröhlich, E. E. et al. Cognitive impairment by antibiotic-induced gut dysbiosis: analysis of gut microbiota-brain communication. Brain. Behav. Immun. 56, 140-155 (2016).

52. Tochitani, S. et al. Administration of non-absorbable antibiotics to pregnant mice to perturb the maternal gut microbiota is associated with alterations in offspring behavior. PLOS ONE 11, e0138293 (2016).

53. Leclercq, S. et al. Low-dose penicillin in early life induces long-term changes in murine gut microbiota, brain cytokines and behavior. Nat. Commun. 8, 15062 (2017).

54. Wang, S. et al. Antibiotic-induced microbiome depletion is associated with resilience in mice after chronic social defeat stress. J. Affect. Disord. 260, 448-457 (2020).

55. Simrén, M. et al. Intestinal microbiota in functional bowel disorders: a Rome foundation report. Gut 62, 159-176 (2013).

56. Mayer, E. A., Savidge, T. \& Shulman, R. J. Brain-gut microbiome interactions and functional bowel disorders. Gastroenterology 146, 1500-1512 (2014). 
57. Gatta, L. \& Scarpignato, C. Mo2055 rifaximin for treatment of small intestine bacterial overgrowth (SIBO): a systematic review and meta-analysis. Gastroenterology 144, S-729 (2013).

58. Calanni, F., Renzulli, C., Barbanti, M. \& Viscomi, G. C. Rifaximin: beyond the traditional antibiotic activity. J. Antibiot. (Tokyo) 67, 667-670 (2014).

59. Shayto, R. H., Abou Mrad, R. \& Sharara, A. I. Use of rifaximin in gastrointestinal and liver diseases. World J. Gastroenterol. 22, 6638-6651 (2016).

60. Descombe, J. J., Dubourg, D., Picard, M. \& Palazzini, E. Pharmacokinetic study of Rifaximin after oral administration in healthy volunteers. Int. J. Clin. Pharmacol. Res. 14, 51-56 (1994).

61. DuPont, H. L. et al. A randomized, double-blind, placebo-controlled trial of Rifaximin to prevent travelers' diarrhea. Ann. Intern. Med. 142, 805-812 (2005).

62. DuPont, H. L. Biologic properties and clinical uses of rifaximin. Expert Opin. Pharmacother. 12, 293-302 (2011).

63. Blandizzi, C., Viscomi, G. C., Marzo, A. \& Scarpignato, C. Is generic rifaximin still a poorly absorbed antibiotic? A comparison of branded and generic formulations in healthy volunteers. Pharmacol. Res. 85, 39-44 (2014).

64. Wang, H., Braun, C. \& Enck, P. Effects of rifaximin on central responses to social stress-a pilot experiment. Neurotherapeutics 15, 807-818 (2018).

65. Craig, A. D. How do you feel? Interoception: the sense of the physiological condition of the body. Nat. Rev. Neurosci. 3, 655-666 (2002).

66. Avery, J. A. et al. A common gustatory and interoceptive representation in the human mid-insula. Hum. Brain Mapp. 36, 2996-3006 (2015).

67. Bowyer, S. M. Coherence a measure of the brain networks: past and present. Neuropsychiatr. Electrophysiol. 2, 1 (2016).

68. Fries, P. Rhythms for cognition: communication through coherence. Neuron 88, 220-235 (2015).

69. Senkowski, D., Schneider, T. R., Foxe, J. J. \& Engel, A. K. Crossmodal binding through neural coherence: implications for multisensory processing. Trends Neurosci. 31, 401-409 (2008).

70. Fries, P. A mechanism for cognitive dynamics: neuronal communication through neuronal coherence. Trends Cognit. Sci. 9, 474-480 (2005).

71. Engel, A. K. \& Fries, P. Beta-band oscillations-signalling the status quo?. Curr. Opin. Neurobiol. 20, 156-165 (2010).

72. Cañas, J., Quesada, J. F., Antolí, A. \& Fajardo, I. Cognitive flexibility and adaptability to environmental changes in dynamic complex problem-solving tasks. Ergonomics 46, 482-501 (2003).

73. Gabrys, R. L., Tabri, N., Anisman, H. \& Matheson, K. Cognitive control and flexibility in the context of stress and depressive symptoms: the cognitive control and flexibility questionnaire. Front. Psychol. 9, 2219 (2018).

74. Koster, E. H. W., De Lissnyder, E., Derakshan, N. \& De Raedt, R. Understanding depressive rumination from a cognitive science perspective: the impaired disengagement hypothesis. Clin. Psychol. Rev. 31, 138-145 (2011).

75. Hofmann, S. G., Sawyer, A. T., Fang, A. \& Asnaani, A. Emotion dysregulation model of mood and anxiety disorders. Depress. Anxiety 29, 409-416 (2012).

76. Gruner, P. \& Pittenger, C. Cognitive inflexibility in obsessive-compulsive disorder. Neuroscience 345, 243-255 (2017).

77. Tchanturia, K. et al. Poor cognitive flexibility in eating disorders: examining the evidence using the wisconsin card sorting task. PLoS ONE 7, e28331 (2012).

78. Lacy, B. E. et al. Bowel disorders. Gastroenterology 150, 1393-1407.e5 (2016).

79. Kassinen, A. et al. The fecal microbiota of irritable bowel syndrome patients differs significantly from that of healthy subjects. Gastroenterology 133, 24-33 (2007).

80. Salonen, A., de Vos, W. M. \& Palva, A. Gastrointestinal microbiota in irritable bowel syndrome: present state and perspectives. Microbiology 156, 3205-3215 (2010).

81. Carroll, I. M., Ringel-Kulka, T., Siddle, J. P. \& Ringel, Y. Alterations in composition and diversity of the intestinal microbiota in patients with diarrhea-predominant irritable bowel syndrome. Neurogastroenterol. Motil. Off. J. Eur. Gastrointest. Motil. Soc. 24, $521-530$ (2012).

82. Jeffery, I. B., Quigley, E. M. M., Öhman, L., Simrén, M. \& O’Toole, P. W. The microbiota link to irritable bowel syndrome. Gut Microbes 3, 572-576 (2012).

83. Pimentel, M. et al. 267 the first large scale deep sequencing of the duodenal microbiome in irritable bowel syndrome reveals striking differences compared to healthy controls. Gastroenterology 144, S59 (2013).

84. Collins, S. M. A role for the gut microbiota in IBS. Nat. Rev. Gastroenterol. Hepatol. 11, 497-505 (2014).

85. Shukla, R., Ghoshal, U., Dhole, T. N. \& Ghoshal, U. C. Fecal microbiota in patients with irritable bowel syndrome compared with healthy controls using real-time polymerase chain reaction: an evidence of dysbiosis. Dig. Dis. Sci. 60, 2953-2962 (2015).

86. Pittayanon, R. et al. Gut microbiota in patients with irritable bowel syndrome-a systematic review. Gastroenterology 157, 97-108 (2019).

87. Whitehead, W. E., Palsson, O. \& Jones, K. R. Systematic review of the comorbidity of irritable bowel syndrome with other disorders: what are the causes and implications?. Gastroenterology 122, 1140-1156 (2002).

88. Fadgyas-Stanculete, M., Buga, A.-M., Popa-Wagner, A. \& Dumitrascu, D. L. The relationship between irritable bowel syndrome and psychiatric disorders: from molecular changes to clinical manifestations. J. Mol. Psychiatry 2, 1-7 (2014).

89. Lee, Y.-T. et al. Risk of psychiatric disorders following irritable bowel syndrome: a nationwide population-based cohort study. PLOS ONE 10, e0133283 (2015).

90. Strunk, D. R., Lopez, H. \& DeRubeis, R. J. Depressive symptoms are associated with unrealistic negative predictions of future life events. Behav. Res. Ther. 44, 861-882 (2006).

91. Murphy, F., Michael, A. \& Sahakian, B. Emotion modulates cognitive flexibility in patients with major depression. Psychol. Med. 42, 1373-1382 (2011).

92. LeMoult, J. \& Gotlib, I. H. Depression: a cognitive perspective. Clin. Psychol. Rev. 69, 51-66 (2019).

93. Lydiard, R. B. Irritable bowel syndrome, anxiety, and depression: what are the links?. J. Clin. Psychiatry 62, 38-45 (2001).

94. Cho, H. S. et al. Anxiety, depression and quality of life in patients with irritable bowel syndrome. Gut Liver 5, 29-36 (2011).

95. Fond, G. et al. Anxiety and depression comorbidities in irritable bowel syndrome (IBS): a systematic review and meta-analysis. Eur. Arch. Psychiatry Clin. Neurosci. 264, 651-660 (2014).

96. Berg, E. A. A simple objective technique for measuring flexibility in thinking. J. Gen. Psychol. 39, 15-22 (1948).

97. Aizawa, E. et al. Altered cognitive function of prefrontal cortex during error feedback in patients with irritable bowel syndrome, based on fMRI and dynamic causal modeling. Gastroenterology 143, 1188-1198 (2012).

98. Wong, K.M.-F. et al. Nature and specificity of altered cognitive functioning in IBS. Neurogastroenterol. Motil. 31, e13696 (2019).

99. Herweg, N. A., Solomon, E. A. \& Kahana, M. J. Theta oscillations in human memory. Trends Cogn. Sci. 24, 208-227 (2020).

100. Fell, J. et al. Rhinal-hippocampal theta coherence during declarative memory formation: interaction with gamma synchronization?. Eur. J. Neurosci. 17, 1082-1088 (2003).

101. Sauseng, P. et al. Theta coupling in the human electroencephalogram during a working memory task. Neurosci. Lett. 354, 123-126 (2004).

102. Summerfield, C. \& Mangels, J. A. Coherent theta-band EEG activity predicts item-context binding during encoding. NeuroImage 24, 692-703 (2005). 
103. Sato, N. \& Yamaguchi, Y. Theta synchronization networks emerge during human object-place memory encoding. NeuroReport 18, 419-424 (2007).

104. Payne, L. \& Kounios, J. Coherent oscillatory networks supporting short-term memory retention. Brain Res. 1247, 126-132 (2009).

105. Fell, J. \& Axmacher, N. The role of phase synchronization in memory processes. Nat. Rev. Neurosci. 12, 105-118 (2011).

106. Burke, J. F. et al. Synchronous and asynchronous theta and gamma activity during episodic memory formation. J. Neurosci. 33, 292-304 (2013).

107. Solomon, E. A. et al. Dynamic theta networks in the human medial temporal lobe support episodic memory. Curr. Biol. 29, 1100-1111.e4 (2019).

108. Payne, J. et al. The impact of stress on neutral and emotional aspects of episodic memory. Memory 14, 1-16 (2006).

109. Payne, J. D. et al. Stress administered prior to encoding impairs neutral but enhances emotional long-term episodic memories. Learn. Mem. 14, 861-868 (2007).

110. Payne, J. D. \& Kensinger, E. A. Stress, sleep, and the selective consolidation of emotional memories. Curr. Opin. Behav. Sci. 19, 36-43 (2018).

111. Gomborone, J. E., Dewsnap, P. A., Libby, G. W. \& Farthing, M. J. Selective affective biasing in recognition memory in the irritable bowel syndrome. Gut 34, 1230-1233 (1993).

112. Seminowicz, D. A. et al. Regional gray matter density changes in brains of patients with irritable bowel syndrome. Gastroenterology 139, 48-57.e2 (2010).

113. Labus, J. et al. Irritable Bowel Syndrome in female patients is associated with alterations in structural brain networks. Pain 155, 137-149 (2014).

114. Icenhour, A. et al. Brain functional connectivity is associated with visceral sensitivity in women with irritable bowel syndrome. NeuroImage Clin. 15, 449-457 (2017).

115. Weng, Y. et al. Disrupted functional connectivity density in irritable bowel syndrome patients. Brain Imaging Behav. 11, 1812$1822(2017)$

116. Weaver, K. R., Sherwin, L. B., Walitt, B., Melkus, G. D. \& Henderson, W. A. Neuroimaging the brain-gut axis in patients with irritable bowel syndrome. World J. Gastrointest. Pharmacol. Ther. 7, 320-333 (2016).

117. Piché, M. et al. Thicker posterior insula is associated with disease duration in women with irritable bowel syndrome (IBS) whereas thicker orbitofrontal cortex predicts reduced pain inhibition in both IBS patients and controls. J. Pain 14, 1217-1226 (2013).

118. Hong, J.-Y. et al. Sex and disease-related alterations of anterior insula functional connectivity in chronic abdominal pain. J. Neurosci. 34, 14252-14259 (2014).

119. Curtis, K. et al. Insular resting state functional connectivity is associated with gut microbiota diversity. Eur. J. Neurosci. 50, 2446-2452 (2019).

120. Pimentel, M., Park, S., Mirocha, J., Kane, S. V. \& Kong, Y. The effect of a nonabsorbed oral antibiotic (rifaximin) on the symptoms of the irritable bowel syndrome. Ann. Intern. Med. 145, 557-563 (2006).

121. Pimentel, M. et al. Rifaximin therapy for patients with irritable bowel syndrome without constipation. N. Engl. J. Med. 364, 22-32 (2011).

122. Lembo, A. et al. T1390 rifaximin for the treatment of diarrhea-associated irritable bowel syndrome: short term treatment leading to long term sustained response. Gastroenterology 134, A545 (2008).

123. Lembo, A. et al. Repeat treatment with rifaximin is safe and effective in patients with diarrhea-predominant irritable bowel syndrome. Gastroenterology 151, 1113-1121 (2016).

124. Menees, S. B., Maneerattannaporn, M., Kim, H. M. \& Chey, W. D. The Efficacy and safety of rifaximin for the irritable bowel syndrome: a systematic review and meta-analysis. Off. J. Am. Coll. Gastroenterol. ACG 107, 28-35 (2012).

125. Yang, J., Lee, H.-R., Low, K., Chatterjee, S. \& Pimentel, M. Rifaximin versus other antibiotics in the primary treatment and retreatment of bacterial overgrowth in IBS. Dig. Dis. Sci. 53, 169-174 (2008).

126. Stinton, L. M. \& Jayakumar, S. Minimal hepatic encephalopathy. Can. J. Gastroenterol. 27, 572-574 (2013).

127. Bajaj, J. S. et al. Rifaximin improves driving simulator performance in a randomized trial of patients with minimal hepatic encephalopathy. Gastroenterology 140, 478-487.e1 (2011).

128. Bajaj, J. S. et al. Modulation of the metabiome by rifaximin in patients with cirrhosis and minimal hepatic encephalopathy. PLoS ONE 8, e60042 (2013).

129. Sanyal, A. et al. Randomised clinical trial: Rifaximin improves health-related quality of life in cirrhotic patients with hepatic encephalopathy-a double-blind placebo-controlled study. Aliment. Pharmacol. Ther. 34, 853-861 (2011).

130. Sidhu, S. et al. Rifaximin improves cognitive functions and health-related quality of life in patients with minimal hepatic encephalopathy: a prospective, randomized, double-blind, placebo-controlled trial (the RIME trial). Gastroenterology 139, e18-e19 (2010).

131. Ahluwalia, V. et al. Enhancement of functional connectivity, working memory and inhibitory control on multi-modal brain MR imaging with rifaximin in cirrhosis: implications for the gut-liver-brain axis. Metab. Brain Dis. 29, 1017-1025 (2014).

132. Bajaj, J. S. et al. Linkage of gut microbiome with cognition in hepatic encephalopathy. Am. J. Physiol. Gastrointest. Liver Physiol. 302, G168-175 (2012).

133. Chey, W. D., Shah, E. D. \& DuPont, H. L. Mechanism of action and therapeutic benefit of rifaximin in patients with irritable bowel syndrome: a narrative review. Ther. Adv. Gastroenterol. 13, 1756284819897531 (2020).

134. Maccaferri, S. et al. Rifaximin modulates the colonic microbiota of patients with Crohn's disease: an in vitro approach using a continuous culture colonic model system. J. Antimicrob. Chemother. 65, 2556-2565 (2010).

135. Ponziani, F. R. et al. The role of antibiotics in gut microbiota modulation: the eubiotic effects of rifaximin. Dig. Dis. 34, 269-278 (2016).

136. Bolling, D. Z. et al. Dissociable brain mechanisms for processing social exclusion and rule violation. NeuroImage 54, 2462-2471 (2011).

137. Bolling, D. Z. et al. Development of neural systems for processing social exclusion from childhood to adolescence. Dev. Sci. 14, 1431-1444 (2011).

138. Williams, K. D. \& Jarvis, B. Cyberball: a program for use in research on interpersonal ostracism and acceptance. Behav. Res. Methods 38, 174-180 (2006).

139. Zalesky, A., Fornito, A. \& Bullmore, E. T. Network-based statistic: identifying differences in brain networks. NeuroImage 53, 1197-1207 (2010)

140. Oostenveld, R., Fries, P., Maris, E. \& Schoffelen, J.-M. FieldTrip: open source software for advanced analysis of MEG, EEG, and invasive electrophysiological data. Comput. Intell. Neurosci. 2011, e156869 (2010).

141. Xia, M., Wang, J. \& He, Y. BrainNet viewer: a network visualization tool for human brain connectomics. PLoS ONE 8, e68910 (2013).

142. FreeSurfer. http://surfer.nmr.mgh.harvard.edu/

143. Dale, A. M., Fischl, B. \& Sereno, M. I. Cortical surface-based analysis I. Segmentation and surface reconstruction. NeuroImage 9, 179-194 (1999) 
144. Fischl, B., Sereno, M. I. \& Dale, A. M. Cortical surface-based analysis. II: inflation, flattening, and a surface-based coordinate system. NeuroImage 9, 195-207 (1999).

145. 5. SUMA - AFNI, SUMA and FATCAT: v20.3.03. https://afni.nimh.nih.gov/pub/dist/doc/htmldoc/SUMA/main_toc.html.

146. Saad, Z. S. \& Reynolds, R. C. SUMA . NeuroImage 62, 768-773 (2012).

147. Li Hegner, Y. et al. Increased functional MEG connectivity as a hallmark of MRI-negative focal and generalized epilepsy. Brain Topogr. 31, 863-874 (2018).

148. Schoffelen, J.-M. \& Gross, J. Source connectivity analysis with MEG and EEG. Hum. Brain Mapp. 30, 1857-1865 (2009).

149. Hillebrand, A. \& Barnes, G. R. A quantitative assessment of the sensitivity of whole-head MEG to activity in the adult human cortex. NeuroImage 16, 638-650 (2002).

150. Piastra, M. C. et al. A comprehensive study on electroencephalography and magnetoencephalography sensitivity to cortical and subcortical sources. Hum. Brain Mapp. https://doi.org/10.1002/hbm.25272 (2020)

151. Network Based Statistic Toolbox-Brain Connectivity Toolbox. https://sites.google.com/site/bctnet/comparison/nbs.

\section{Author contributions}

H.W., C.Br. and P.E. designed the study. H.W. setup the experiment and acquired the neuromagnetic data. D.S. and C.Ba. performed the network analysis of the neuromagnetic data. C.Br. was involved in the data analysis as consultant. D.S. and C.Ba. has written the manuscript. All authors were involved in the discussion of the experiment and reviewed the manuscript.

\section{Funding}

Open Access funding enabled and organized by Projekt DEAL.

\section{Competing interests}

The authors declare no competing interests.

\section{Additional information}

Supplementary Information The online version contains supplementary material available at https://doi.org/ 10.1038/s41598-021-83994-4.

Correspondence and requests for materials should be addressed to D.S.

Reprints and permissions information is available at www.nature.com/reprints.

Publisher's note Springer Nature remains neutral with regard to jurisdictional claims in published maps and institutional affiliations.

(c) (i) Open Access This article is licensed under a Creative Commons Attribution 4.0 International License, which permits use, sharing, adaptation, distribution and reproduction in any medium or format, as long as you give appropriate credit to the original author(s) and the source, provide a link to the Creative Commons licence, and indicate if changes were made. The images or other third party material in this article are included in the article's Creative Commons licence, unless indicated otherwise in a credit line to the material. If material is not included in the article's Creative Commons licence and your intended use is not permitted by statutory regulation or exceeds the permitted use, you will need to obtain permission directly from the copyright holder. To view a copy of this licence, visit http://creativecommons.org/licenses/by/4.0/.

(c) The Author(s) 2021 